\title{
SUMS OF MULTIPLICATIVE FUNCTIONS OVER A BEATTY SEQUENCE
}

\author{
AHMET M. GÜLOĞLU ${ }^{\bowtie}$ and C. WESLEY NEVANS
}

(Received 3 March 2008)

\begin{abstract}
We study sums involving multiplicative functions that take values over a nonhomogenous Beatty sequence. We then apply our result in a few special cases to obtain asymptotic formulas for quantities such as the number of integers in a Beatty sequence that are representable as a sum of two squares up to a given magnitude.
\end{abstract}

2000 Mathematics subject classification: 11E25, 11B83.

Keywords and phrases: sums of multiplicative functions, Beatty sequences.

\section{Introduction}

Let $A \geq 1$ be an arbitrary constant, and let $\mathcal{F}_{A}$ be the set of multiplicative functions $f$ such that $|f(p)| \leq A$ for all primes $p$ and

$$
\sum_{n \leq N}|f(n)|^{2} \leq A^{2} N \quad(N \in \mathbb{N}) .
$$

Exponential sums of the form

$$
S_{\alpha, f}(N)=\sum_{n \leq N} f(n) e(n \alpha) \quad\left(\alpha \in \mathbb{R}, f \in \mathcal{F}_{A}\right),
$$

where $e(z)=e^{2 \pi i z}$ for $z \in \mathbb{R}$, occur frequently in analytic number theory. Montgomery and Vaughan have shown (see [8, Corollary 1]) that the upper bound

$$
S_{\alpha, f}(N) \ll_{A} \frac{N}{\log N}+\frac{N(\log R)^{3 / 2}}{R^{1 / 2}}
$$

holds uniformly for all $f \in \mathcal{F}_{A}$, provided that $|\alpha-a / q| \leq q^{-2}$ with some reduced fraction $a / q$ for which $2 \leq R \leq q \leq N / R$. In this paper, we use the MontgomeryVaughan result to estimate sums of the form

(c) 2008 Australian Mathematical Society 0004-9727/08 \$A2.00+0.00 


$$
G_{\alpha, \beta, f}(N)=\sum_{\substack{n \leq N \\ n \in \mathcal{B}_{\alpha, \beta}}} f(n),
$$

where $\alpha, \beta \in \mathbb{R}$ with $\alpha>1, f \in \mathcal{F}_{A}$, and $\mathcal{B}_{\alpha, \beta}$ is the nonhomogenous Beatty sequence defined by

$$
\mathcal{B}_{\alpha, \beta}=\{n \in \mathbb{N}: n=\lfloor\alpha m+\beta\rfloor \text { for some } m \in \mathbb{Z}\} .
$$

Our results are uniform over the family $\mathcal{F}_{A}$ and nontrivial whenever

$$
\lim _{N \rightarrow \infty} \frac{\log N}{N \log \log N}\left|\sum_{n \leq N} f(n)\right|=\infty,
$$

a condition which guarantees that the error term in Theorem 1 is smaller than the main term. One can remove this condition, at the expense of losing uniformity with respect to $f$, and still obtain Theorem 1 for any bounded arithmetic function $f$ (not necessarily multiplicative) for which the exponential sums in (2) satisfy

$$
S_{\alpha, f}(N)=o\left(\sum_{n \leq N} f(n)\right) \quad(N \rightarrow \infty) .
$$

The general problem of characterizing functions for which this relation holds appears to be rather difficult; see [1] for Bachman's conjecture and his related work on this problem.

We shall also assume that $\alpha$ is irrational and of finite type $\tau$. For an irrational number $\gamma$, the type of $\gamma$ is defined by

$$
\tau=\sup \left\{t \in \mathbb{R}: \liminf _{n \rightarrow \infty} n^{t} \llbracket \gamma n \rrbracket=0\right\},
$$

where $\llbracket \cdot \rrbracket$ denotes the distance to the nearest integer. Dirichlet's approximation theorem implies that $\tau \geq 1$ for every irrational number $\gamma$. According to theorems of Khinchin [6] and Roth [10], $\tau=1$ for almost all real numbers (in the sense of the Lebesgue measure) and all irrational algebraic numbers $\gamma$, respectively; also see $[2,11]$.

Our main result is the following theorem.

THEOREM 1. Let $\alpha, \beta \in \mathbb{R}$ with $\alpha>1$, and suppose that $\alpha$ is irrational and of finite type. Then, for all $f \in \mathcal{F}_{A}$,

$$
G_{\alpha, \beta, f}(N)=\alpha^{-1} \sum_{n \leq N} f(n)+O\left(\frac{N \log \log N}{\log N}\right),
$$

where the implied constant depends only on $\alpha$ and A.

The following corollaries are immediate applications of Theorem 1. 
COROLlary 2. The number of integers not exceeding $N$ that lie in the Beatty sequence $\mathcal{B}_{\alpha, \beta}$ and can be represented as a sum of two squares is

$$
\#\left\{n \leq N: n \in \mathcal{B}_{\alpha, \beta}, n=\square+\square\right\}=\frac{C N}{\alpha \sqrt{\log N}}+O\left(\frac{N \log \log N}{\log N}\right),
$$

where

$$
C=2^{-1 / 2} \prod_{p \equiv 3}\left(1-p^{-2}\right)^{-1 / 2}=0.76422365 \ldots
$$

is the Landau-Ramanujan constant.

To state the next result, we recall that an integer $n$ is said to be $k$-free if $p^{k} \nmid n$ for every prime $p$.

COROLlaRY 3. For every $k \geq 2$, the number of $k$-free integers not exceeding $N$ that lie in the Beatty sequence $\mathcal{B}_{\alpha, \beta}$ is

$$
\#\left\{n \leq N: n \in \mathcal{B}_{\alpha, \beta}, n \text { is } k \text {-free }\right\}=\alpha^{-1} \zeta^{-1}(k) N+O\left(\frac{N \log \log N}{\log N}\right),
$$

where $\zeta(s)$ is the Riemann zeta function.

Finally, we consider the average value of the number of representations of an integer from a Beatty sequence as a sum of four squares.

COROLLARY 4. Let $r_{4}(n)$ denote the number of representations of $n$ as a sum of four squares. Then

$$
\sum_{\substack{n \leq N \\ n \in \mathcal{B}_{\alpha, \beta}}} r_{4}(n)=\frac{\pi^{2} N^{2}}{2 \alpha}+O\left(\frac{N^{2} \log \log N}{\log N}\right),
$$

where the implied constant depends only on $\alpha$.

Any implied constants in the symbols $O$ and $\ll$ may depend on the parameters $\alpha$ and $A$ but are absolute otherwise. We recall that the notation $X \ll Y$ is equivalent to $X=O(Y)$.

\section{Preliminaries}

2.1. Discrepancy of fractional parts We define the discrepancy $D(M)$ of a sequence of real numbers $b_{1}, b_{2}, \ldots, b_{M} \in[0,1)$ by

$$
D(M)=\sup _{\mathcal{I} \subseteq[0,1)}\left|\frac{\mathcal{V}(\mathcal{I}, M)}{M}-\right| \mathcal{I}||,
$$

where the supremum is taken over all possible subintervals $\mathcal{I}=(a, c)$ of the interval $[0,1), \mathcal{V}(\mathcal{I}, M)$ is the number of positive integers $m \leq M$ such that $b_{m} \in \mathcal{I}$, and $|\mathcal{I}|=c-a$ is the length of $\mathcal{I}$. 
If an irrational number $\gamma$ is of finite type, we let $D_{\gamma, \delta}(M)$ denote the discrepancy of the sequence of fractional parts $(\{\gamma m+\delta\})_{m=1}^{M}$. By [7, Theorem 3.2, Ch. 2], we have the following result.

LEMMA 5. For a fixed irrational number $\gamma$ of finite type $\tau$ and for all $\delta \in \mathbb{R}$,

$$
D_{\gamma, \delta}(M) \leq M^{-1 / \tau+o(1)} \quad(M \rightarrow \infty)
$$

where the function defined by $o(\cdot)$ depends only on $\gamma$.

2.2. Numbers in a Beatty sequence The following result is standard in characterizing the elements of the Beatty sequence $\mathcal{B}_{\alpha, \beta}$.

LEMMA 6. Let $\alpha, \beta \in \mathbb{R}$ with $\alpha>1$, and set $\gamma=\alpha^{-1}$ and $\delta=\alpha^{-1}(1-\beta)$. Then $n=\lfloor\alpha m+\beta\rfloor$ for some $m \in \mathbb{Z}$ if and only if $0<\{\gamma n+\delta\} \leq \gamma$.

From Lemma 6, an integer $n$ lies in $\mathcal{B}_{\alpha, \beta}$ if and only if $n \geq 1$ and $\psi(\gamma n+\delta)=1$, where $\psi$ is the periodic function with period one whose values on the interval $(0,1]$ are given by

$$
\psi(x)= \begin{cases}1 & \text { if } 0<x \leq \gamma \\ 0 & \text { if } \gamma<x \leq 1 .\end{cases}
$$

We wish to approximate $\psi$ by a function whose Fourier series representation is well behaved. This will give rise to the aforementioned exponential sum $S_{\alpha, f}(N)$. To this end, we use the result of Vinogradov (see [15, Ch. I, Lemma 12]) which states that for any $\Delta$ such that

$$
0<\Delta<\frac{1}{8} \quad \text { and } \quad \Delta \leq \frac{1}{2} \min \{\gamma, 1-\gamma\}
$$

there exists a real-valued function $\Psi$ with the following properties:

(i) $\Psi$ is periodic with period one;

(ii) $0 \leq \Psi(x) \leq 1$ for all $x \in \mathbb{R}$;

(iii) $\Psi(x)=\psi(x)$ if $\Delta \leq\{x\} \leq \gamma-\Delta$ or if $\gamma+\Delta \leq\{x\} \leq 1-\Delta$;

(iv) $\Psi$ can be represented by a Fourier series

$$
\Psi(x)=\sum_{k \in \mathbb{Z}} g(k) \mathbf{e}(k x),
$$

where $g(0)=\gamma$ and the Fourier coefficients satisfy the uniform bound

$$
g(k) \ll \min \left\{|k|^{-1},|k|^{-2} \Delta^{-1}\right\} \quad(k \neq 0) .
$$

\section{Proofs}

3.1. Proof of Theorem 1 Using Lemma 6, we rewrite the sum (4) in the form

$$
G_{\alpha, \beta, f}(N)=\sum_{n \leq N} f(n) \psi(\gamma n+\delta) .
$$


Replacing $\psi$ by $\Psi$, we obtain

$$
G_{\alpha, \beta, f}(N)=\sum_{n \leq N} f(n) \Psi(\gamma n+\delta)+O\left(\sum_{n \in V(\Delta, N)} f(n)\right),
$$

where $V(\Delta, N)$ is the set of positive integers $n \leq N$ for which

$$
\{\gamma n+\delta\} \in[0, \Delta) \cup(\gamma-\Delta, \gamma+\Delta) \cup(1-\Delta, 1) .
$$

Since the length of each interval above is at most $2 \Delta$, it follows from definition (6) and Lemma 5 that

$$
|V(\Delta, N)| \ll \Delta N+N^{1-1 /(2 \tau)},
$$

where we have used the fact that $\alpha$ and $\gamma$ have the same type $\tau$. Thus, taking (1) into account, Cauchy's inequality gives

$$
\begin{aligned}
\left|\sum_{n \in V(\Delta, N)} f(n)\right| & \leq|V(I, N)|^{1 / 2}\left(\sum_{n \leq N}|f(n)|^{2}\right)^{1 / 2} \\
& \ll\left((\Delta N)^{1 / 2}+N^{1 / 2-1 /(4 \tau)}\right) N^{1 / 2} \\
& =\Delta^{1 / 2} N+N^{1-1 /(4 \tau)} .
\end{aligned}
$$

Next, let $K \geq \Delta^{-1}$ be a large real number (to be specified later), and let $\Psi_{K}$ be the trigonometric polynomial given by

$$
\Psi_{K}(x)=\sum_{|k| \leq K} g(k) \mathbf{e}(k x)=\gamma+\sum_{0<|k| \leq K} g(k) \mathbf{e}(k x) \quad(x \in \mathbb{R}) .
$$

Using (7), we see that the estimate

$$
\Psi(x)=\Psi_{K}(x)+O\left(K^{-1} \Delta^{-1}\right)
$$

holds uniformly for all $x \in \mathbb{R}$; therefore,

$$
\sum_{n \leq N} f(n) \Psi(\gamma n+\delta)=\sum_{n \leq N} f(n) \Psi_{K}(\gamma n+\delta)+O\left(K^{-1} \Delta^{-1} N\right),
$$

where we have used the bound $\sum_{n \leq N}|f(n)| \ll N$ which follows from (1).

Combining (8), (9), (10) and (11), we derive that

$$
G_{\alpha, \beta, f}(N)=\gamma \sum_{n \leq N} f(n)+H(N)+O\left(K^{-1} \Delta^{-1} N+\Delta^{1 / 2} N+N^{1-1 /(4 \tau)}\right),
$$

where

$$
H(N)=\sum_{0<|k| \leq K} g(k) \mathbf{e}(k \delta) S_{k \gamma, f}(N) .
$$


Put $R=(\log N)^{3}$. We claim that if $N$ is sufficiently large, then for every $k$ in the above sum there is a reduced fraction $a / q$ such that $|k \gamma-a / q| \leq q^{-2}$ and $R \leq q \leq N / R$. Assuming this is true for the moment, (3) implies that

$$
S_{k \gamma, f}(N) \ll \frac{N}{\log N} \quad(0<|k| \leq K) ;
$$

using (7), we then deduce that

$$
H(N) \ll \frac{N \log K}{\log N} .
$$

Therefore,

$$
G_{\alpha, \beta, f}(N)-\gamma \sum_{n \leq N} f(n) \ll \frac{N \log K}{\log N}+K^{-1} \Delta^{-1} N+\Delta^{1 / 2} N+N^{1-1 / 4 \tau} .
$$

To balance the error terms, we choose

$$
\Delta=(\log N)^{-2} \text { and } K=\Delta^{-3 / 2}=(\log N)^{3},
$$

thus obtaining the bound stated in the theorem.

To prove the claim, let $k$ be an integer with $0<|k| \leq K=(\log N)^{3}$, and let $r_{i}=a_{i} / q_{i}$ be the $i$ th convergent in the continued fraction expansion of $k \gamma$. Since $\gamma$ is of finite type $\tau$, for every $\varepsilon>0$ there is a constant $C=C(\gamma, \varepsilon)$ such that

$$
C\left(|k| q_{i-1}\right)^{-(\tau+\varepsilon)}<\llbracket \gamma|k| q_{i-1} \rrbracket \leq|\gamma| k\left|q_{i-1}-a_{i-1}\right| \leq q_{i}^{-1} .
$$

Put $\varepsilon=\tau$, and let $j$ be the least positive integer for which $q_{j} \geq R$ (note that $j \geq 2$ ). Then

$$
R \leq q_{j} \ll\left(|k| q_{i-1}\right)^{2 \tau} \leq(K R)^{2 \tau}=(\log N)^{6 \tau},
$$

and it follows that $R \leq q_{j} \leq N / R$ if $N$ is sufficiently large, depending only on $\alpha$. This concludes the proof.

3.2. Proof of Corollary 2 Let $f(n)$ be the characteristic function of the set of integers that can be represented as a sum of two squares. It follows from [4, Theorem 366] that $f(n)$ is multiplicative. Hence Corollary 2 is an immediate consequence of Theorem 1 and the asymptotic formula

$$
\sum_{n \leq N} f(n)=\frac{C N}{(\log N)^{1 / 2}}+O\left(\frac{N}{(\log N)^{3 / 2}}\right)
$$

(see, for example, $[12,13])$, where $C$ is given by (5).

3.3. Proof of Corollary 3 Fix $k \geq 2$ and let $f(n)$ be the characteristic function of the set of $k$-free integers. It is easily proved that $f(n)$ is multiplicative. Thus Corollary 3 follows from Theorem 1 and the following estimate of Gegenbauer [3] for the number of $k$-free integers not exceeding $N$ :

$$
\sum_{n \leq N} f(N)=\zeta^{-1}(k) N+O\left(N^{1 / k}\right)
$$


3.4. Proof of Corollary 4 Put $f(n)=r_{4}(n) /(8 n)$. From Jacobi's formula for $r_{4}(n)$, namely

$$
r_{4}(n)=8\left(2+(-1)^{n}\right) \sum_{\substack{d \mid n \\ d \text { odd }}} d \quad(n \geq 1),
$$

it follows that $f(n)$ is multiplicative and that $f(p) \leq 3 / 2$ for every prime $p$. Moreover, using the formula of Ramanujan [9] (see also [14]),

$$
\sum_{n \leq N} \sigma^{2}(n)=\frac{5}{6} \zeta(3) N^{3}+O\left(N^{2}(\log N)^{2}\right)
$$

where $\sigma$ is the sum of divisors function, we obtain

$$
\sum_{n \leq N}|f(n)|^{2} \leq \sum_{n \leq N} \frac{\sigma^{2}(n)}{n^{2}}=\frac{5}{2} \zeta(3) N+O\left((\log N)^{3}\right)
$$

by partial summation. Therefore, $f(n) \in \mathcal{F}_{A}$ for some constant $A \geq 1$. Applying Theorem 1, we deduce that

$$
\sum_{\substack{n \leq N \\ n \in \mathcal{B}_{\alpha, \beta}}} \frac{r_{4}(n)}{n}=\alpha^{-1} \sum_{n \leq N} \frac{r_{4}(n)}{n}+O\left(\frac{N \log \log N}{\log N}\right),
$$

where the implied constant depends only on $\alpha$.

From the asymptotic formula

$$
\sum_{n \leq N} r_{4}(n)=\frac{\pi^{2} N^{2}}{2}+O(N \log N)
$$

(see for example [5, p. 22]), partial summation gives

$$
\sum_{n \leq N} \frac{r_{4}(n)}{n}=\pi^{2} N+O\left((\log N)^{2}\right) .
$$

Consequently,

$$
\sum_{\substack{n \leq N \\ n \in \mathcal{B}_{\alpha, \beta}}} \frac{r_{4}(n)}{n}=\alpha^{-1} \pi^{2} N+O\left(\frac{N \log \log N}{\log N}\right) .
$$

Using partial summation once more, we obtain the statement of Corollary 4.

\section{Acknowledgements}

We would like to thank William Banks, Pieter Moree and Igor Shparlinski for their helpful comments and careful reading of the original manuscript. 


\section{References}

[1] G. Bachman, 'On exponential sums with multiplicative coefficients, II', Acta Arith. 106(1) (2003), 41-57.

[2] Y. Bugeaud, Approximation by Algebraic Numbers, Cambridge Tracts in Mathematics, 160 (Cambridge University Press, Cambridge, 2004).

[3] L. Gegenbauer, 'Asymptotische Gesetze der Zahlentheori', Denkschirften Akad. Wien 49(1) (1885), 37-80.

[4] G. Hardy and E. Wright, An Introduction to the Theory of Numbers, 5th edn (Oxford University Press, New York, 2005).

[5] H. Iwaniec and E. Kowalski, Analytic Number Theory, American Mathematical Society Colloquium Publications, 53 (American Mathematical Society, Providence, RI, 2004).

[6] A. Y. Khinchin, 'Zur metrischen Theorie der diophantischen approximationen', Math. Z. 24(4) (1926), 706-714.

[7] L. Kuipers and H. Niederreiter, Uniform Distribution of Sequences, Pure and Applied Mathematics (Wiley-Interscience, New York, 1974).

[8] H. L. Montgomery and R. C. Vaughan, 'Exponential sums with multiplicative coefficients', Invent. Math. 43(1) (1977), 69-82.

[9] S. Ramanujan, 'Some formulae in the analytic theory of numbers', Messenger Math. 45 (1916), 81-84.

[10] K. F. Roth, 'Rational approximations to algebraic numbers', Mathematika 2 (1955), 1-20; 'Corrigendum', Mathematika 2 (1955), 168.

[11] W. M. Schmidt, Diophantine Approximation, Lecture Notes in Mathematics, 785 (Springer, Berlin, 1980).

[12] J. P. Serre, 'Divisibilité de certaines fonctions arithmétiques', Enseign. Math. (2) 22(3-4) (1976), 227-260.

[13] D. Shanks, 'The second-order term in the asymptotic expansion of $B(x)$ ', Math. Comp. 18(85) (1964), 75-86.

[14] R. A. Smith, 'An error term of Ramanujan', J. Number Theory 2 (1970), 91-96.

[15] I. M. Vinogradov, The Method of Trigonometrical Sums in the Theory of Numbers (Dover Publications, Inc., Mineola, NY, 2004).

AHMET M. GÜLOĞLU, Department of Mathematics, Bilkent University, Bilkent 06800, Ankara, Turkey

e-mail: guloglua@fen.bilkent.edu.tr

C. WESLEY NEVANS, Department of Mathematics, University of Missouri, Columbia, MO 65211, USA

e-mail: nevans@math.missouri.edu 